\title{
Diagnostic Pharmacokinetics: how brain-derived biomarkers distribute through the human body, and how this affects their diagnostic significance - the case of S100B
}

\section{Robert Murcko}

FloTBI Inc. Cleveland

Nicola Marchi

Institute of Functional Genomics (UMR 5203 CNRS - U 1191 INSERM, University of Montpellier)

Damian Bailey

University of South Wales

Damir Janigro ( $\square$ djanigro@flocel.com )

FloTBI Inc. Cleveland

\section{Research Article}

Keywords: computer model, MATLAB, Simbiology, astrocytes, physiologically-based pharmacokinetic model, glymphatics, extracranial sources, traumatic brain injury, brain barriers, saliva

Posted Date: January 19th, 2022

DOI: https://doi.org/10.21203/rs.3.rs-1277222/v1

License: (c) (i) This work is licensed under a Creative Commons Attribution 4.0 International License.

Read Full License 


\section{Abstract}

Blood biomarkers of neurological diseases are often employed to rule out or confirm the presence of significant intracranial or cerebrovascular pathology or for the differential diagnosis of conditions with similar presentations (e.g., hemorrhagic vs. embolic stroke). More widespread utilization of biomarkers related to brain health is hampered by our incomplete understanding of the kinetic properties, release patterns, and excretion of molecules derived from the brain. This is, in particular, true for S100B, an astrocyte-derived protein released across the blood-brain barrier (BBB). We developed an open-source pharmacokinetic computer model that allows investigations of a biomarker's movement across the body, the sources of a biomarker's release, and its elimination. This model was derived from a general in silico model of drug pharmacokinetics adapted for protein biomarkers. We improved the model's predictive value by adding realistic blood flow values, organ levels of S100B, lymphatic and glymphatic circulation, and glomerular filtration for excretion in urine. Three key variables control biomarker levels in blood or saliva: blood-brain barrier permeability, the S100B partition into peripheral organs, and the cellular levels of S100B in astrocytes. A small contribution to steady-state levels of glymphatic drainage was also observed; this mechanism contributed to the uptake of organs of circulating S100B. Additionally, this open-source model can also mimic the kinetic behavior of other markers, such as GFAP or NF-L. Our results show that $\mathrm{S} 100 \mathrm{~B}$, after uptake by various organs from the systemic circulation, can be released back into systemic fluids at levels that do not significantly affect the clinical significance of venous blood or salivary levels after an episode of BBB disruption.

\section{Background}

Pharmacokinetic in silico models are essential for pharmacological studies and drug development. During the drug discovery and development process, potential clinical candidates are screened for their absorption, distribution, metabolism, and excretion (ADME) properties to avoid clinical failures related to inappropriate ADME properties. Until recently, most pharmacokinetic models were aimed at predicting the properties of small ( $<1 \mathrm{kD}$ ) molecules after oral or intravenous (IV) administration. Recently, biologics have become a significant portion of therapeutic agents, and the old small molecule software strategies had to be reformulated to adapt to larger ( $>10 \mathrm{kD})$ molecular weight proteic therapeutics. While ad hoc software has been developed by Industry, academic efforts have used available platforms (e.g., MATLAB) to model how drugs distribute through the body.

A perhaps unexpected utilization of pharmacokinetic modeling of large, proteic agents is the development of modified strategies to study the movement of diagnostic molecules in the human body. Several of these biomarkers are proteins with varying molecular properties and sizes. These biomarkers are commonly released or synthesized ex novo by a specific organ, neoplasm, or cell type. For example, troponins are proteins found in skeletal and cardiac muscle fibers that regulate muscular contraction. Troponin tests measure the level of cardiac-specific troponin in the blood to help detect heart injury (1). 
When there is damage to heart muscle cells, troponin is released into the blood, thus becoming detectable by a simple blood test. The necessity of cellular death for a biomarker's release is not universal, since many other biomarkers are released by healthy cells (see below for S100B). Our previous work has focused primarily on brain-derived diagnostic molecules used to diagnose CNS or neurological diseases. These include GFAP, S100B, UCHL-1, and other less-studied reporters of brain disease or health.

We adapted and refined MATLAB-based models (3-5) for the present study using the published data obtained by real-life experiments (direct measurements of S100B from human tissues; (2-4)). An example of how pharmacokinetic models can be applied to brain diagnostic markers was published (2). We specifically wished to explore the pharmacokinetics of S100B, a reporter of blood-brain barrier (BBB) dysfunction (BBBD) and brain health (5) (6-9). While several studies have promoted its use in neurology and psychiatry (2, 8-10), others expressed doubts about its reliability for human diagnostics (11). These concerns primarily derive from the pitfalls listed below.

It was suggested that S100B not only derives from the brain but also has extracranial sources (11-16). Thus, when both brain and peripheral trauma are involved, it is impossible to dissect out a central vs. peripheral origin of the biomarker. This is a pitfall in studies where $\mathrm{S100B}$ is elevated in individuals with multi-trauma of orthopedic nature (17). The same issues were reported for other brain-derived biomarkers (18). Several counterarguments have been made, showing, for example, that extracranial sources where $\mathrm{S} 100 \mathrm{~B}$ is synthesized from mRNA are few (e.g., testis, descending tubules in kidney (2)) and that S100B content in other organs derives from uptake from circulation (3). It was recently shown that time-dependent internalization of circulating S100B by mesenchymal stem cells occurs via the pathways of clathrin- and lipid raft-mediated endocytosis (19). Others have demonstrated that S100B in fat tissue does not contribute to peripherally detected levels (20), but the opposite was also suggested (21). Thus, controversy exists regarding the extent and relevance of extracranial sources of biomarkers used for CNS diagnostics.

In the field of sports medicine, it was shown that blood S100B increases after sub-concussive head hits $(22,23)$ : this was ascribed to increased BBB permeability as also documented by MRI $(24)$. Other studies have shown that $S 100 B$ is increased by exercise alone $(13,25,26)$, while others found no effect of strenuous exercise on S100B levels (27-30). An explanation of these contrasting findings points to BBB damage induced by extreme exercise (31). According to this hypothesis, strenuous exercise or performance in extreme sports results in BBB "opening," possibly due to a mechanism involving free radical formation, as suggested by ref. (32). In any case, it is not known how different sources of $\mathrm{S} 100 \mathrm{~B}$ contribute to the peripheral signal in blood (or saliva) $(26,31,33,34)$. Lastly, a common motif in S100B diagnostics is that $\mathrm{S100B}$ is not specific for any neurological disease (2). This is due to the fact that BBB 
leakage allowing S100B appearance in peripheral body fluids is a common feature of many neurological conditions (2).

Another point of contention relates to how the brain releases S100B during an insult. The leading hypothesis calls for a breach of the BBB as described above and in $(35,36)$. An alternative hypothesis calls for the recently described glymphatic circulation as a means of brain release of biomarkers in blood (37). The contribution of glymphatics in human subjects is unknown.

The scope of the present work is to answer, when possible, these questions by using two advanced fullbody models of cerebrovascular and peripheral circulation after the release of S100B by the brain or other organs. A lymphatic compartment was also added to the model together with realistic urinary excretion pathways. The initial parameters of the model were derived from experimental observations and available human data (2).

\section{Methods}

We used MATLAB 2019-2021b (MathWorks, Natick, MA) to design, test or simulate the model. The toolbox used was MATLAB's Simbiology app (versions 5.0 to 6.2), aided by packages for partial differential equations, statistics, and parallel computing. Data were plotted in MATLAB and exported to CorelDraw as extended metafiles (Corel Co.).

We developed two separate and independent models to mimic the behavior of circulating brain-derived small molecular weight proteic biomarkers. Model 1 was used primarily to assess the relevance of various peripheral organs to the signal measured in blood. Model 2 was developed after ruling out the contributions by heart, bone, and skin (Figure 3): these organs are not included in Model 2, which uses a different set of equations to focus on the contributions of adipose, muscle, and gut tissues to measured levels of the biomarker in blood. Model 2 also adopts a more complex brain modeling paradigm, as detailed below. The following sections highlight the shared and specific modeling strategies used.

Most of the simulations shown were run to steady-state with or without an accompanying BBB disruption event. This allows investigators to follow up the kinetics of $\mathrm{S100B}$ in each organ or compartment For Figure 10, the BBBD was triggered after steady-state was achieved.

Model 1 
A human full-body physiologically-based pharmacokinetic (PBPK) model was adapted from (38). This model contains lung, brain, skin, bone, adipose tissue, heart, kidney, muscle, and gut compartments (Figure 1). The volume of these organs is specified in Table 1. The organs are connected by arterial and venous vessels, whose contributions to the vascular network are expressed in $\mathrm{ml} / \mathrm{hr}$ and listed in Table 1. No data are available on their role related to the release and uptake of S100B or other markers of brain health. It was shown that the spleen contains $\mathrm{S} 100 \mathrm{~B}$, but this expression was limited to $\mathrm{CD} 4^{+} / \mathrm{CD} 8^{+}$ immunocompetent cells (3).

The initial levels of S100B $(\mathrm{ng} / \mathrm{ml})$ in each organ were derived from our previous work based on actual measurements $(2,3)$. Each organ in the model contains a vascular fraction, i.e., an interface between parenchyma and vascular space (Figure 2). The volume of the vascular fraction was obtained from (38). The circulation arterial-venous loop did not involve the heart and pulmonary circulation but rather consisted of a path through the lung (Figure 1). A lymphatic circulatory system was added to all tissues; we modeled a central lymph collection where each lymphatic vessel out of tissue collects before drainage into venous blood. The volumes of the organs and circulatory districts modeled herein were obtained from $(2,39)$ and are reported in Table 1.

The primary source of S100B in the body is the brain (40). In our model, brain release of S100B into circulation is controlled by the variable $B B B_{-} I n d e x$. This dimensionless value varies from 0 to 1 (except Figure 10), reflecting no permeability across an intact BBB or "BBB opening," respectively.

Eq. 1

$\mathrm{d}($ Brain.S100B $) / \mathrm{dt}=$ 1/Brain* $\left(\left(\left(\mathrm{kf} \_\right.\right.\right.$brain*Brain.S100B-kr_brain*[Brain tissue vascular fraction].S100B)*interstitialFlow*BBB_Index))

where $k f_{-} b r a i n$ and $k r_{-} b r a i n$ are dimensionless constants obtained based on the two-pore model as per references $(38,41)$. Due to their size and polarity, protein biomarkers have limited direct diffusion across endothelial cell membranes. The fluid and protein movement occurs mainly by diffusion and convection through pores in the endothelial wall, which is limited by protein size. Data sources were gathered from (38) to determine $\mathrm{kr}$ and $\mathrm{kf}$ values for model 1 . Small pore radii and large pore radii values for various tissue types were noted. Additionally, the ratio of small pore count to large pore count for that tissue type was noted, also provided in (38). 
Using the data gathered, a ratio was taken to determine the magnitude of differences between the total amount of large pore radii within a tissue versus the total amount of small pore radii within a tissue. The equation used was:

Eq. $1 \mathrm{~A}$

kr_organ $=(($ Large Pore Radius Size $) /($ Small Pore Radius Size*Ratio of Small Pores to Large Pores $))$.

To create a more pronounced differential within each tissue, but keep the ratio of $k$ values between each tissue standardized, the $\mathrm{kf}$ value was the $\mathrm{kr}$ value multiplied by a factor of 10,000 , via kf_organ = kr_organ $\star 10,000$. The general equation for the organ's uptake or release of protein biomarkers was:

Eq. 2

$\mathrm{d}($ Organ.S100B) $/ \mathrm{dt}=1 /$ Organ*(-((kf_Organ*Organ.S100B-kr_Organ*[Organ Tissue Vascular Fraction].S100B)*interstitialFlow))

where the value Interstitial flow represents the flow rate of the protein within the organ, Organ and Tissue Vascular Fraction volumes were derived from ref. (38).

Excretion of the biomarker protein was modeled by kidney filtration:

Eq. 3

$\mathrm{d}($ Urine.S100B $) / \mathrm{dt}=1 /$ Urine ${ }^{\star}\left(\left(\right.\right.$ RenalEliminationFactor ${ }^{\star}($ GFR/Kidney $) \star$ Kidney.S100B $) \star$ Kidney $\left.)\right)$

where GFR is the glomerular filtration rate, and the Renal elimination factor is an additional dimensionless tuning parameter ranging from 0 to 1 . 
Sensitivity analysis is the study of how the uncertainty in the output of a mathematical model can be divided and allocated to different sources of uncertainty in its inputs. In Simbiology, the routine of sensitivity analysis allows determining which rate constants and concentrations in a model significantly influence the overall behavior of the model. SimBiology supports two types of sensitivity analyses: local and global sensitivity analysis. We used global sensitivity analysis to interpret the impact of S100B in various organs on venous and arterial biomarker levels (Figure 3). Sensitivity analyses were run with $B B B_{-}$Index set to either 0 or 1 (Figures $3 C$ and 3D, respectively). Full dedimensionalization was used for sensitivity analysis to compare the contributions of different species to the output to be analyzed (typically venous blood levels of S100B).

Model 2

Model 2 follows the general structural backbone of Model 1 (Figure 4). However, organs (except for the kidney, see below) are subdivided into vascular and interstitial compartments (Table 2). To describe the passage of protein from the interstitial (parenchymal) space into vascular space, we used the coefficient of vascular reflection (Sigma, or s) as per reference (42). The size-dependent restriction of large pores and small pores can, in fact, be represented as the vascular reflection coefficient, an indirect measure of the density of exchange pores. The model used to mimic the brain (Figure 5) used BBB_Index and Trauma_Index variables to describe the passage of $\mathrm{S100B}$ across the interstitial, vascular, and cellular compartments. Note that unlike the dimensionless rate constants in Model 1, kinetic variables have dimensions of quantity/time in this model. The equation governing changes of biomarker's levels in the vascular compartment was:

\section{Eq. 4}

$\mathrm{d}([$ Vascular Compartment Brain].S100B)/dt)] = 1/[Vascular Compartment Brain]* ((ArterialToBrainBloodFlow*[Arterial Blood].S100B) - (BrainToVenousBloodFlow*[Vascular Compartment Brain].S100B $)+\left(\left(B B B \_I n d e x *[\right.\right.$ Interstitium Brain].S100B $) *[$ Interstitium Brain] $\left.)\right)$

where $B B B_{-} I n d e x$ can change between 0 and 1 to mimic increased permeability of the cerebral vasculature. In addition to having a three-compartment structure, brain modeling also included glymphatic drainage into central lymph and venous blood. The equation for brain interstitium S100B was thus: 
Eq. 5

$\mathrm{d}([$ Interstitium Brain].S100B $) / \mathrm{dt}=1 /$ [Interstitium Brain $] *(($ Trauma_Index*Glia.S100B $) \star$ Glia $)$ (Glymphatics*[Interstitium Brain].S100B) - ((BBB_Index*[Interstitium Brain].S100B)*[Interstitium Brain]))

where the term Trauma_Index refers to the passage of S100B from astrocytes in the cellular compartment (Glia) released directly into the brain interstitium. Glymphatics is the rate of interstitial flow to Central lymph. Changes of S100B in the cellular compartment were described by:

Eq. 6

[Interstitium Brain].S100B=[Glia.S100B]Trauma_Index

Glia.S100B was set constant at $50 \mathrm{ng} / \mathrm{ml}$ as per in vitro measurements by others (43).

The kidney was modeled by a single compartment with input from arterial blood and an output to urine. The process was described by:

Eq. 7

$\mathrm{d}\left(\right.$ Kidney.S100B) $/ \mathrm{dt}=1 /$ Kidney*$^{*}-(($ EliminationFactor* (GFR/Kidney)*Kidney.S100B/TissuePartitionKidney)*Kidney) + (ArterialToKidneyBloodFlow*[Arterial Blood].S100B) - (KidneyToVenousBloodFlow*Kidney.S100B))

and by:

Eq. 8

$\mathrm{d}($ Urine.S100B $) / \mathrm{dt}=1 /$ Urine $^{\star}((($ EliminationFactor $*$

(GFR/Kidney)*Kidney.S100B/TissuePartitionKidney)*Kidney) - (kf*Urine.S100B)) 
Tissue partition kidney was set at $0.1 /$ minute. GFR was set at 10 milliliter/hour, although a non-constant value explored during simulations (e.g., Figure 8).

Note that in all figures, except Figure 10, the simulation started before steady-state conditions were reached, thus allowing the variables to express the kinetic significance of the underlying code. See, for example, Figure 3AB, where the time-dependent changes in S100B are shown.

\section{Results}

The structure of Model 1 is shown in Figure 1, together with the graphic rendition of the process of BBB disruption. Figure 2 shows the formalism used to describe each organ in Model 1. The simulation of Model 1 led to the results shown in Figure 3, which represents the changes in organs' S100B levels under normal conditions (Figure $3 \mathrm{~A}$ ) or after BBB disruption (Figure 3B; $B B B_{-}$Index=1). Without BBB disruption, individual organs displayed a change in parenchymal $5100 \mathrm{~B}$ content to reach steady-state eventually. Also, note that venous levels, at steady-state, were $<0.1 \mathrm{ng} / \mathrm{ml}$, which is consistent with clinical studies in normal adults when using the Roche Diagnostics test $(44,45)$. Panels $C$ and $D$ show the results of the simulation in A and B processed for sensitivity analysis (see Methods). The main contributors to venous blood levels were muscle and gut tissues, with minor contributions by adipose, lung, and skin. After BBB disruption, sensitivity analysis pointed to brain sources as primary contributors to venous levels.

Since only a few organs contributed significantly to the overall venous signal, we developed Model 2 based on three organs (muscle, adipose, and gut) plus the kidney and a "virtual" urine container mimicking the bladder (Figure 4). Salivary production was also added to model 2. A key difference between the two models is the description of brain S100B movements within and outside the brain parenchyma. For the brain in Model 2, three compartments were used: vascular (i.e., the cerebrovascular circulation), interstitial (the brain extracellular space), and glia, referring to astrocytes, which are the primary cell type expressing S100B in the body (Figure 5). The correspondence of the model with brain physiology is shown in Figure 5B. In addition to the arterial influx and venous efflux, a glymphatic distribution process draining into Central lymph was added to the model. Another difference in Model 2 is that the structure of the organs and S100B movements within was based on the reflection coefficient (Sigma) rather than two-pore theory calculations (see Methods).

We ran a sensitivity analysis for steady-state values of Central lymph, Arterial blood S100B, and Venous blood S100B within Model 2. Under normal conditions $\left(B B B_{-} I n d e x=0\right)$, the main contributor to the peripheral fluid signals was gut S100B (Figure 6). When glymphatics were added to the simulation, the brain contribution to the S100B signal surpassed the gut. When BBB disruption was simulated 
( $\left.B B B_{-} I n d e x=1\right)$, the main contributor to the signal in blood remained the brain, but the contribution of gut levels affected Central lymph readouts. In addition to BBB disruption, we simulated brain trauma (opening the communication between the glial content of $\mathrm{S100B}$ with the brain interstitium): during the simulation, brain interstitial S100B remained the chief contributor to the vascular levels of S100B. The profiles of venous changes under these conditions are shown in Figure 7A. Note the small but measurable contribution of glymphatic drainage to the venous signal.

We previously measured S100B in several peripheral organs (see $(2,3))$ and assigned these values as initial conditions for the simulations presented herein (see Tables 1 and 2). We tested the hypothesis that the levels measured in peripheral organs lacking mRNA for S100B were due to diffusion of S100B from the blood. Figure 7B shows these changes. We started the initial conditions with all organ levels set arbitrarily at 0 to test the extent of organs' uptake of circulating S100B. Note (Figure 7B1) the increase in S100B due to the vascular uptake over a long period of control conditions $\left(B B B_{-}\right.$Index $\left.=0\right)$. Figure 7B2 shows the contribution to the venous levels of glymphatics and when the $B B B_{-} I n d e x$ is set to 0 . The data in Figure $\mathrm{C} 1-\mathrm{C} 3$ show the changes in variables when BBBD and trauma are present. We studied the changes in several compartments (Figure $7 \mathrm{C}$ ) under the same conditions. Note the effects of BBB disruption (C2) and trauma (C3) to organs and blood. Thus, peripheral organs take up S100B from the circulation to, in turn, contribute to blood levels.

The impact of glomerular filtration rate (GFR) and urine formation on blood S100B levels was evaluated in Model 2 (Figure 8). Under intact or BBBD conditions, GFR greatly influenced the levels of S100B measured in blood, lymphatics, urine, and organs. When setting GFR to zero (Figure 8A), we found a profound effect of kidney excretion on both organ (left panel) and biological fluids (right panel). In Figure 8B, GFR was set at 10 or 100 while also varying BBB_Index from 0 to 1.

Recent reports used salivary S100B and compared its values to venous blood levels (34). We simulated the passive extravasation of arterial blood to form crevicular fluid (46), see Figure 9. The levels of S100B in saliva, at steady-state, were larger than those in blood when blood flow to saliva was adjusted to $4 \mathrm{ml}$ (upper end of physiologic levels (47)).

We formulated the hypothesis that after BBB disruption the half-life of S100B in blood is determined in part by the availability of $\mathrm{S100B}$ in the brain interstitium. This was tested as follows (Figure 10). We simulated a BBBD after reaching steady-state at two time points (arrows in Figure 10). Note that a broad range of $B B B_{-} I n d e x$ was explored (indicated in Figure 10). Also, note that when the Trauma_Index was 0 , the second BBBD episode had little effect on S100B, unless the first BBBD was minimal (0.1). We then 
repeated the simulation with Trauma_Index set to 1 . The secondary BBBD response was restored parallel to a decreased depletion of interstitial S100B in the brain (not shown). This suggests that levels of S100B in the interstitium of the brain are in part responsible for the time-dependent changes in S100B in blood.

\section{Discussion}

The simulation efforts presented herein revealed several surprising findings requiring human trials or animal experiments to be confirmed or refuted. These unexpected results are listed in separate paragraphs below.

\section{Extracranial sources}

It is well known that the distribution of $\mathrm{S100B}$ protein is not restricted to the brain. Several extracranial sources have been hypothesized to contribute to the blood levels used clinically $(3,4,14,15,18)$. Our simulation revealed that the contribution of "usual suspects" skin, and adipose tissue is relatively minor compared to the impact of gut and muscle release of S100B (Figures 3 and 6). The possible explanation of these levels of S100B may depend on either local synthesis or uptake from blood. Since mRNA for $\mathrm{S} 100 \mathrm{~B}$ is lacking in gut tissue (3), the second explanation was tested (Figure 7B1) by running a simulation where initial values for organs' S100B were arbitrarily set to zero while allowing for glymphatic-mediated contribution to blood (see also below). At steady state, peripheral tissues were loaded with levels of S100B comparable to those measured in vivo (Figure 7A, B1) (2). While the effects of glymphatics on venous levels was small (7A), a prolonged stimulation (100 hours) unveiled a powerful effect on organs' levels of S100B (7B1), suggesting that glymphatic connection between brain and periphery was sufficient to load previously depleted organs with the biomarker. Note (Figure 7B2) that when organs' levels, BBBD, and glymphatics were set to 0 , no venous signal was seen, suggesting that these three parameters are the exclusive contributors to steady-state organs' levels of S100B. Thus, the most parsimonious explanation for the peripheral presence of S100B is the uptake of circulating protein, as also shown in an animal model (3). Conversely, these levels remain relatively stable once achieved until an event, such as BBB disruption occurs (Figure 7C1-3).

The question of whether peripheral levels of $\mathrm{S100B}$ contribute to the venous signal was answered by simulating control conditions or by adding BBB disruption events (Figure 7A). At pre-BBBD time points, the brain influenced the blood signal via a mechanism involving glymphatic circulation (see above). BBBD (Figure 7C2) and the trauma index in Figure 7C3 dwarfed the control changes in S100B caused by other sources. Sensitivity analysis showed that gut and muscle, but not kidney or adipose tissue, influence venous levels pre-BBBD, but brain contribution dominates after BBB disruption. Our results have thus shown that circulating S100B released by glymphatics in lymph and venous fluids is a likely 
scenario explaining tissue levels in the absence of transcription. Additional BBB disruption does not increase S100B in organs (Figure 7C1-3). We have also shown that serum levels of S100B are only marginally affected by the release of S100B from organs, since the increased venous S100B never approached the cut-off value of 0.1 or 0.15 nanogram/milliliter, which is the clinical ceiling for controls $(44,48)$.

\section{Do glymphatics contribute to biomarker blood levels?}

It was suggested that the primary source of S100B after traumatic brain injury is the brain's communication with blood via glymphatic drainage (37). We found no significant contribution of glymphatics to the overall signal in blood after BBBD (Figure 6, and Figure 7C1-C3)). However, a small steady-state contribution of glymphatics to the pre-BBBD signal was observed (Figure 7A). This implies a continuous "trickle" of brain protein from the extracellular space into the blood via lymphatic drainage under physiological conditions. If this were the case, as also shown by us (32), one expects that levels in blood will continuously increase, which is not what has been shown in human subjects. A fraction of what is being released from the brain is taken up by peripheral tissues as discussed above, but the factor that fully counteracts this constant source of S100B is kidney excretion of S100B (Figure 8). In fact, when GFR was set to zero (in A), a constant increase was observed in peripheral fluids and organs. Thus, an equilibrium exists between glomerular filtration of small molecular weight protein (2) and S100B release from the brain interstitium via the glymphatic system. This finding predicts that patients with reduced glomerular filtration rate may have elevated levels of $\mathrm{S100B}$ (and other biomarkers) in the absence of a BBB contribution.

\section{Effects of parenchymal trauma on biomarker's levels}

Brain damage and BBB disruption contribute to the overall levels of S100B in blood $(35,36)$. However, in clinical practice it is impossible to dissect out the contributions of these two factors independently. We have developed a subroutine in our software model that allows us to quantify and describe these two sources of blood S100B (Figure 10). We ran a simulation where levels of blood S100B in response to two BBB disruption events were monitored. When the Trauma_index was set to zero (no contribution of cellular release of S100B on peripheral or brain interstitial levels), we noted that the second BBB disruption episode did not cause an increase of $S 100 B$ in venous blood unless a minimal level of disruption $\left(B B B_{-}\right.$Index $\left.=0.1\right)$ was used for the first event. We monitored the reserve of S100B sources in the interstitium to show that depletion of interstitial S100B occurred after the first, more significant, episodes. Therefore, the subsequent BBB "opening" was consequential only if a minimal depletion of S100B occurred. When the Trauma_Index was set to 1, replenishment of S100B in the extracellular space of the brain was repristinated, allowing for $\mathrm{S100B}$ release after the second BBB disruption event. This is a 
potentially important finding since it suggests that astrocytic sources of S100B are crucial in controlling the extent and duration of S100B during BBB disruptions.

\section{Comparison with existing models of blood biomarkers}

In addition to our own prototype model $(2,33,49)$, an effort to mimic biomarkers' fate after TBI has been published (50). The Authors use a much simplified, one-compartment model derived from oral absorption of therapeutic drugs. The limitations we found to be most relevant compared to the present study are: 1) Lack of distribution variables. Because only one compartment is used, the marker undergoes only blood distribution and thus ignores extracranial sources or the uptake of the marker by organs. 2) The model has only one path for the biomarker to leave the brain, ignoring glymphatic drainage. 3) Being a single-compartment model, there is no effort to reproduce organ size (including the brain) or cerebral and organ blood flow. 4) The excretion data are presented as a means to balance brain release.

\section{Limitations}

The main limitation of our study is that we did not attempt to adapt the model to existing data on S100B, except when using realistic quantities of S100B in peripheral organs and a comparison of data with a previously established control value ceiling. We also used blood flow and volumetric data from the literature and accepted equilibrium values derived from the simulation of protein distribution after local injection (38). Therefore, our modeling effort was not geared toward reproducing existing data but rather to allow for a discovery process of mechanisms that human subject-derived data make impossible to study.

\section{Future directions}

The open-source format of the software developed herein (available at FloTBI.com) will enable other researchers to adapt the core model to other situations and answer questions perhaps related to other biomarkers. Future developments will add the effect of molecular size (molecular weight and radius; see (2)) on the movement across different compartments. This was already done in an older version of this model (2). The main changes due to molecular size are likely to affect kidney filtration, passage across the BBB, and the overall kinetic properties of the marker. As new markers of CNS function are unveiled, we will focus on the physical and chemical properties of these proteins related to the voyage across organs and biofluids. In addition, we will develop a model that considers other biological variables, such as sex and age (2). Finally, the model based on human subjects can be allometrically manipulated to include laboratory animals which are often used as surrogate experimental targets. 


\section{Declarations}

All manuscripts must contain the following sections under the heading 'Declarations':

- Ethics approval and consent to participate NA

- Consent for publication All Authors agreed to publish this manuscript

- Availability of data and materials The program will be posted as open-source on the internet

- Competing interests DJ has shares in FloTBI, a company specializing in salivary biomarkers of brain dysfunction.

- Funding NONE

- Authors' contributions DJ and RM performed the simulations and built the program and participated to writing and editing of the manuscript.

- NM and DB provided data to develop the models used and participated to writing and editing of the manuscript.

- Acknowledgements NONE

- Authors' information (optional)

\section{References}

1. Segraves JM, Frishman WH. Highly sensitive cardiac troponin assays: a comprehensive review of their clinical utility. Cardiol Rev. 2015;23(6):282-9.

2. Dadas A, Washington J, Marchi N, Janigro D. Improving the clinical management of traumatic brain injury through the pharmacokinetic modeling of peripheral blood biomarkers. Fluids Barriers CNS. 2016;13(1):21.

3. Bargerstock E, Puvenna V, Iffland P, Falcone T, Hossain M, Vetter S, et al. Is peripheral immunity regulated by blood-brain barrier permeability changes? PLoS One. 2014;9(7):e101477.

4. Pham N, Fazio V, Cucullo L, Teng Q, Biberthaler P, Bazarian JJ, et al. Extracranial Sources of S100B Do Not Affect Serum Levels. Plos One. 2010;5(9):e12691.

5. Preston E, Webster J, Small D. Characteristics of sustained blood-brain barrier opening and tissue injury in a model for focal trauma in the rat. J Neurotrauma. 2001;18(1):83-92.

6. Kanner AA, Marchi N, Fazio V, Mayberg MR, Koltz MT, Siomin V, et al. Serum S100beta: a noninvasive marker of blood-brain barrier function and brain lesions. Cancer. 2003;97(11):2806-13.

7. Marchi N, Rasmussen P, Kapural M, Fazio V, Kight K, Mayberg MR, et al. Peripheral markers of brain damage and blood-brain barrier dysfunction. Restor Neurol Neurosci. 2003;21(3-4):109-21. 
8. Kanner AA, Marchi N, Fazio V, Mayberg MR, Koltz MT, Siomin V, et al. Serum S100beta: a noninvasive marker of blood-brain barrier function and brain lesions. Cancer. 2003;97(11):2806-13.

9. Marchi N, Cavaglia M, Bhudia S, Hallene K, Janigro D. Peripheral markers of blood-brain barrier damage. Clinica Chimica Acta. 2004;342((1-2)):1-12.

10. Falcone T, Fazio V, Lee C, Simon B, Franco K, Marchi N, et al. Serum S100B: a potential biomarker for suicidality in adolescents? PLoS One. 2010;5(6):e11089.

11. Papa L, Silvestri S, Brophy GM, Giordano P, Falk JL, Braga CF, et al. GFAP out-performs S100beta in detecting traumatic intracranial lesions on computed tomography in trauma patients with mild traumatic brain injury and those with extracranial lesions. J Neurotrauma. 2014;31(22):1815-22.

12. Bouzat P, Francony G, Declety P, Brun J, Kaddour A, Renversez JC, et al. Can serum protein S100 beta predict neurological deterioration after moderate or minor traumatic brain injury? Annales Francaises D Anesthesie et de Reanimation. 2009;28(2):135-9.

13. Hasselblatt M, Mooren FC, von Ahsen N, Keyvani K, Fromme A, Schwarze-Eicker K, et al. Serum S100beta increases in marathon runners reflect extracranial release rather than glial damage. Neurology. 2004;62(9):1634-6.

14. Korfias S, Stranjalis G, Papadimitriou A, Psachoulia C, Daskalakis G, Antsaklis A, et al. Serum S100B protein as a biochemical marker of brain injury: a review of current concepts. Curr Med Chem. 2006;13(30):3719-31.

15. Savola O, Pyhtinen J, Leino TK, Siitonen S, Niemela O, Hillbom M. Effects of head and extracranial injuries on serum protein S100B levels in trauma patients. J Trauma. 2004;56(6):1229-34.

16. Thelin EP, Jeppsson E, Frostell A, Svensson M, Mondello S, Bellander BM, et al. Utility of neuronspecific enolase in traumatic brain injury; relations to S100B levels, outcome, and extracranial injury severity. Crit Care. 2016;20:285.

17. Anderson RE, Hansson LO, Nilsson O, Dijlai-Merzoug R, Settergren G. High serum S100B levels for trauma patients without head injuries. Neurosurgery. 2001;48(6):1255-8.

18. Posti JP, Hossain I, Takala RS, Liedes H, Newcombe V, Outtrim J, et al. Glial Fibrillary Acidic Protein and Ubiquitin C-Terminal Hydrolase-L1 Are Not Specific Biomarkers for Mild CT-Negative Traumatic Brain Injury. J Neurotrauma. 2017.

19. Zhang Y, Zhu J, Xu H, Yi Q, Yan L, Ye L, et al. Time-Dependent Internalization of S100B by Mesenchymal Stem Cells via the Pathways of Clathrin- and Lipid Raft-Mediated Endocytosis. Front Cell Dev Biol. 2021;9:674995. 
20. Pham N, Fazio V, Cucullo L, Teng Q, Biberthaler P, Bazarian JJ, et al. Extracranial sources of S100B do not affect serum levels. PLoS One. 2010;5(9).

21. Steiner J, Schiltz K, Walter M, Wunderlich MT, Keilhoff G, Brisch R, et al. S100B serum levels are closely correlated with body mass index: an important caveat in neuropsychiatric research.

Psychoneuroendocrinology. 2010;35(2):321-4.

22. Kawata K, Rubin LH, Takahagi M, Lee JH, Sim T, Szwanki V, et al. Subconcussive Impact-Dependent Increase in Plasma S100beta Levels in Collegiate Football Players. J Neurotrauma. 2017;34(14):2254-60.

23. Zonner SW, Ejima K, Bevilacqua ZW, Huibregtse ME, Charleston C, Fulgar C, et al. Association of Increased Serum S100B Levels With High School Football Subconcussive Head Impacts. Front Neurol. 2019;10:327.

24. Tagge CA, Fisher AM, Minaeva OV, Gaudreau-Balderrama A, Moncaster JA, Zhang XL, et al. Concussion, microvascular injury, and early tauopathy in young athletes after impact head injury and an impact concussion mouse model. Brain. 2018;141(2):422-58.

25. Schulte S, Podlog LW, Hamson-Utley JJ, Strathmann FG, Struder HK. A systematic review of the biomarker S100B: implications for sport-related concussion management. J Athl Train. 2014;49(6):83050 .

26. Michetti F, Bruschettini M, Frigiola A, Abella R, Giamberti A, Marchese N, et al. Saliva S100B in professional sportsmen: High levels at resting conditions and increased after vigorous physical activity. Clin Biochem. 2011;44(2-3):245-7.

27. Watson P, Shirreffs SM, Maughan RJ. Blood-brain barrier integrity may be threatened by exercise in a warm environment. Am J Physiol Regul Integr Comp Physiol. 2005;288(6):R1689-R94.

28. Watson P, Black KE, Clark SC, Maughan RJ. Exercise in the heat: effect of fluid ingestion on bloodbrain barrier permeability. Med Sci Sports Exerc. 2006;38(12):2118-24.

29. Schulte S, Schiffer T, Sperlich B, Kleinoder H, Holmberg HC. Serum Concentrations of S100B are not Affected by Cycling to Exhaustion With or Without Vibration. J Hum Kinet. 2011;30:59-63.

30. Koh SX, Lee JK. S100B as a marker for brain damage and blood-brain barrier disruption following exercise. Sports Med. 2014;44(3):369-85.

31. Janigro D, Bailey DM, Lehmann S, Badaut J, O'Flynn R, Hirtz C, et al. Peripheral Blood and Salivary Biomarkers of Blood-Brain Barrier Permeability and Neuronal Damage: Clinical and Applied Concepts. Front Neurol. 2020;11:577312.

32. Bailey DMea. Hypoxemia promotes blood-brain barrier destabilization of the neurovascular unit during extreme apnea in humans. Journal of Cerebral Blood Flow \& Metabolism. 2022;2022 in press. 
33. Dadas A, Janigro D. The role and diagnostic significance of cellular barriers after concussive head trauma. Concussion. 2018;3(1):Cnc53.

34. Janigro D, Kawata K, Silverman E, Marchi N, Diaz-Arrastia R. Is Salivary S100B a Biomarker of Traumatic Brain Injury? A Pilot Study. Front Neurol. 2020;11:528.

35. Marchi N, Cavaglia M, Fazio V, Bhudia S, Hallene K, Janigro D. Peripheral markers of blood-brain barrier damage. Clin Chim Acta. 2004;342(1-2):1-12.

36. Marchi N, Rasmussen PA, Kapural M, Fazio V, Cavaglia M, Janigro D. Peripheral markers of brain damage and blood-brain barrier dysfunction. Restorative Neurology and Neuroscience. 2003;21(3-4):10921.

37. Plog BA, Dashnaw ML, Hitomi E, Peng WG, Liao YH, Lou NH, et al. Biomarkers of Traumatic Injury Are Transported from Brain to Blood via the Glymphatic System. Journal of Neuroscience. 2015;35(2):518-26.

38. Gill KL, Gardner I, Li L, Jamei M. A Bottom-Up Whole-Body Physiologically Based Pharmacokinetic Model to Mechanistically Predict Tissue Distribution and the Rate of Subcutaneous Absorption of Therapeutic Proteins. AAPS J. 2016;18(1):156-70.

39. Peters SA. Evaluation of a generic physiologically based pharmacokinetic model for lineshape analysis. Clin Pharmacokinet. 2008;47(4):261-75.

40. Heizmann CW, Fritz G, Schafer BW. S100 proteins: Structure, functions and pathology. Frontiers in Bioscience. 2002;7:D1356-D68.

41. Sepp A, Meno-Tetang G, Weber A, Sanderson A, Schon O, Berges A. Computer-assembled crossspecies/cross-modalities two-pore physiologically based pharmacokinetic model for biologics in mice and rats. J Pharmacokinet Pharmacodyn. 2019;46(4):339-59.

42. Shah DK, Betts AM. Towards a platform PBPK model to characterize the plasma and tissue disposition of monoclonal antibodies in preclinical species and human. J Pharmacokinet Pharmacodyn. 2012;39(1):67-86.

43. Morquette P, Verdier D, Kadala A, Fethiere J, Philippe AG, Robitaille R, et al. An astrocyte-dependent mechanism for neuronal rhythmogenesis. Nat Neurosci. 2015;18(6):844-54.

44. Iverson GL, Posti JP, Ohman J, Blennow K, Zetterberg H, Luoto TM. Reliability of serum S100B measurement following mild traumatic brain injury: a comparison of assay measurements from two laboratories. Brain Inj. 2020;34(9):1237-44.

45. Bouvier D, Duret $T$, Rouzaire P, Jabaudon $M$, Rouzaire $M$, Nourrisson $C$, et al. Preanalytical, analytical, gestational and pediatric aspects of the S100B immuno-assays. Clin Chem Lab Med. 
2016;54(5):833-42.

46. Rahim MA, Rahim ZH, Ahmad WA, Hashim OH. Can Saliva Proteins Be Used to Predict the Onset of Acute Myocardial Infarction among High-Risk Patients? Int J Med Sci. 2015;12(4):329-35.

47. Jasim H, Olausson P, Hedenberg-Magnusson B, Ernberg M, Ghafouri B. The proteomic profile of whole and glandular saliva in healthy pain-free subjects. Sci Rep. 2016;6:39073.

48. Okonkwo DO, Puffer RC, Puccio AM, Yuh EL, Yue JK, Diaz-Arrastia R, et al. Point-of-Care Platform Blood Biomarker Testing of Glial Fibrillary Acidic Protein versus S100 Calcium-Binding Protein B for Prediction of Traumatic Brain Injuries: A Transforming Research and Clinical Knowledge in Traumatic Brain Injury Study. J Neurotrauma. 2020.

49. Dadas A, Washington J, Diaz-Arrastia R, Janigro D. Biomarkers in traumatic brain injury (TBI): a review. Neuropsychiatr Dis Treat. 2018;14:2989-3000.

50. Azizi S, Hier DB, Allen B, Obafemi-Ajayi T, Olbricht GR, Thimgan MS, et al. A Kinetic Model for Blood Biomarker Levels After Mild Traumatic Brain Injury. Front Neurol. 2021;12:668606.

\section{Tables}

Tables 1-2 are in the supplementary files section.

\section{Figures}



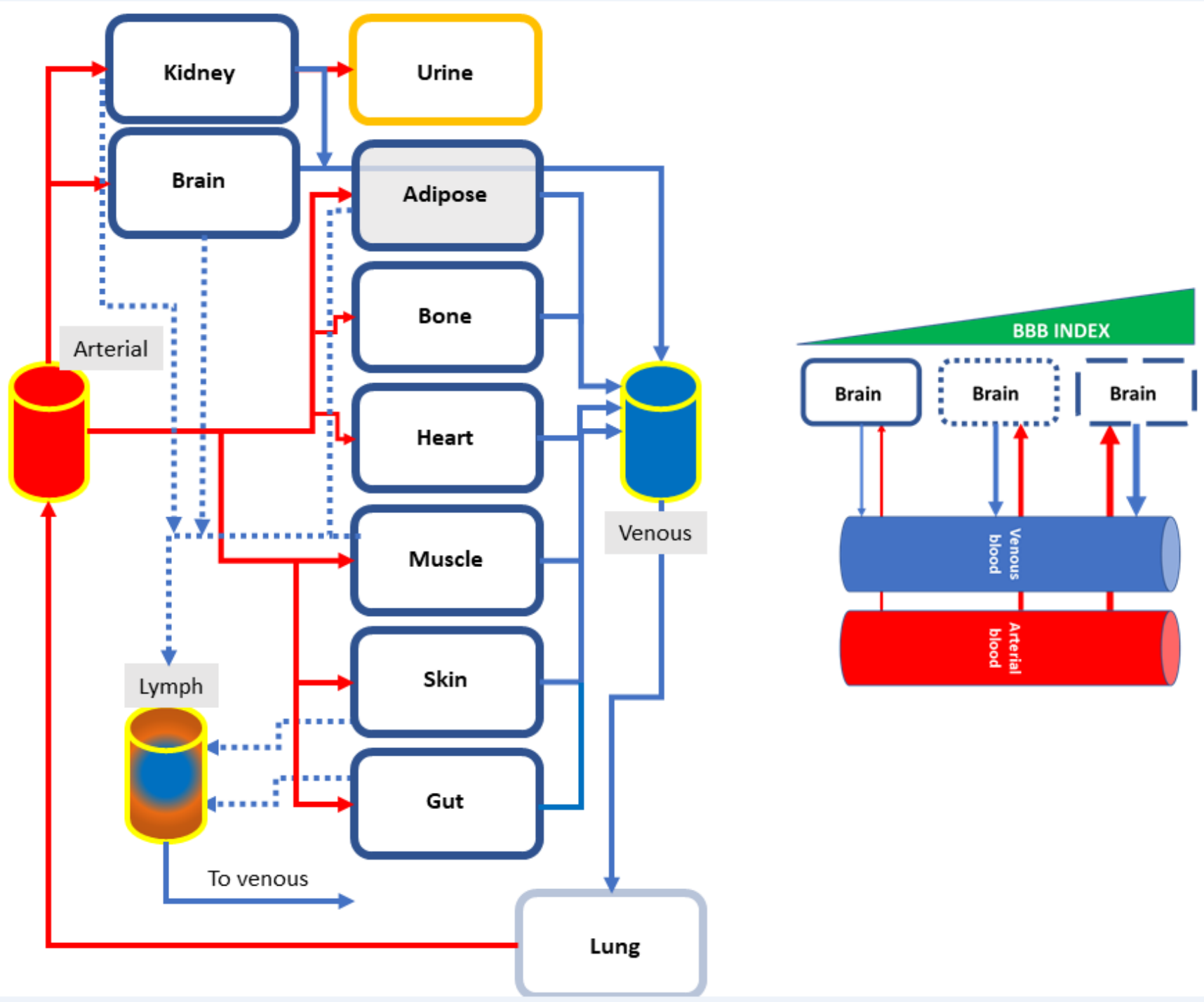

\section{Figure 1}

Schematic representation of the structure of Model 1. The continuous red lines depict flow through arteries (except for the lung), while the continuous blue lines refer to venous flow. The dotted blue lines show the lymphatic vessels connecting the organs directly to the venous compartment. The right panel inset shows a graphical representation of the mechanism of BBBD presented herein, underscoring that venous levels of S100B are greatly influenced by leakage of biomarkers from the brain into the circulation. 


\section{Organ}

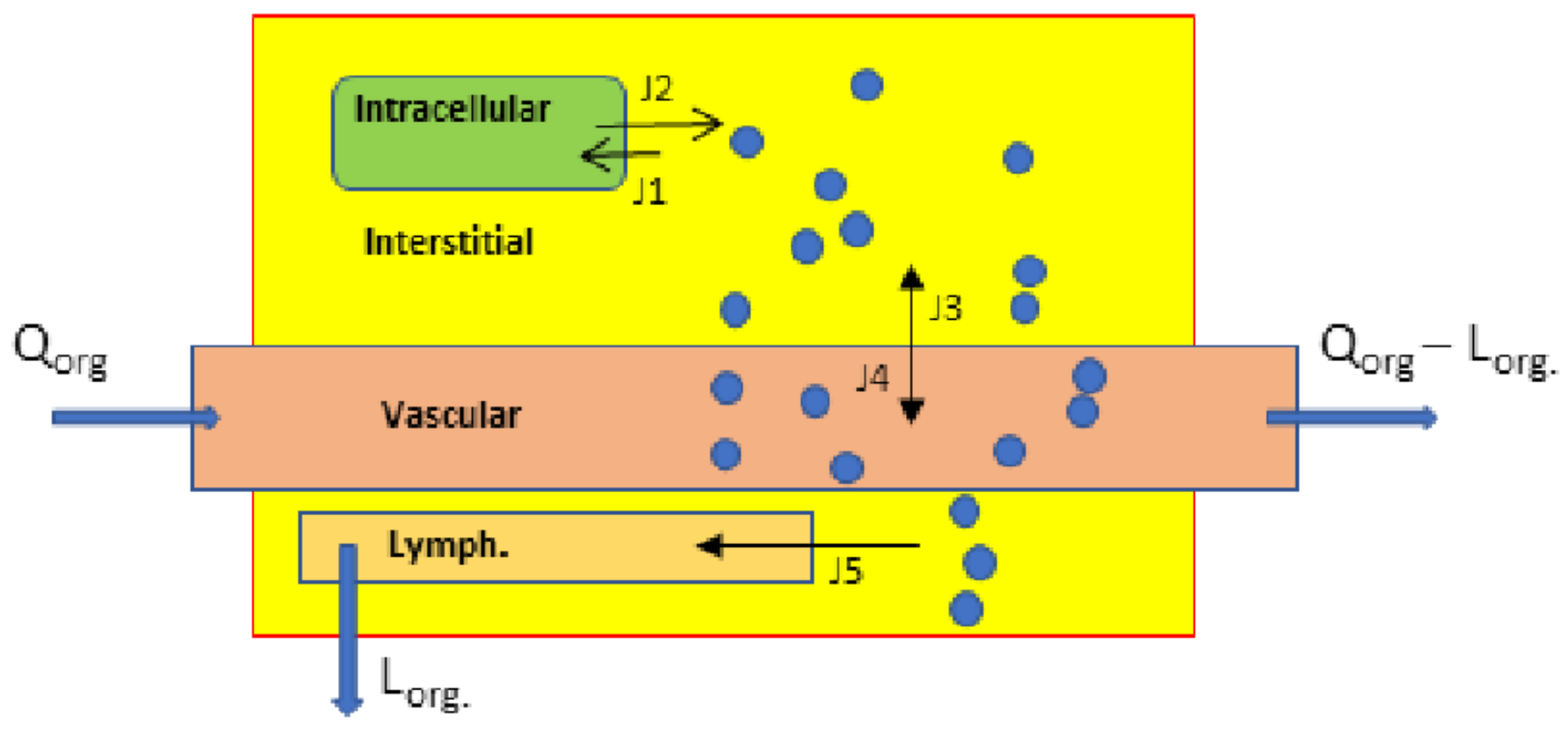

Figure 2

Structure of a single organ in Model 1. Note that two equilibria reactions $\left(K_{r}\right.$ and $\left.K_{f}\right)$ describe the passage of biomarkers from the organ's parenchyma to blood and vice versa. A lymphatic vessel is also depicted. Qorg and Lorg refer to the blood flow into and out of the organ, and lymphatic flow, respectively. 


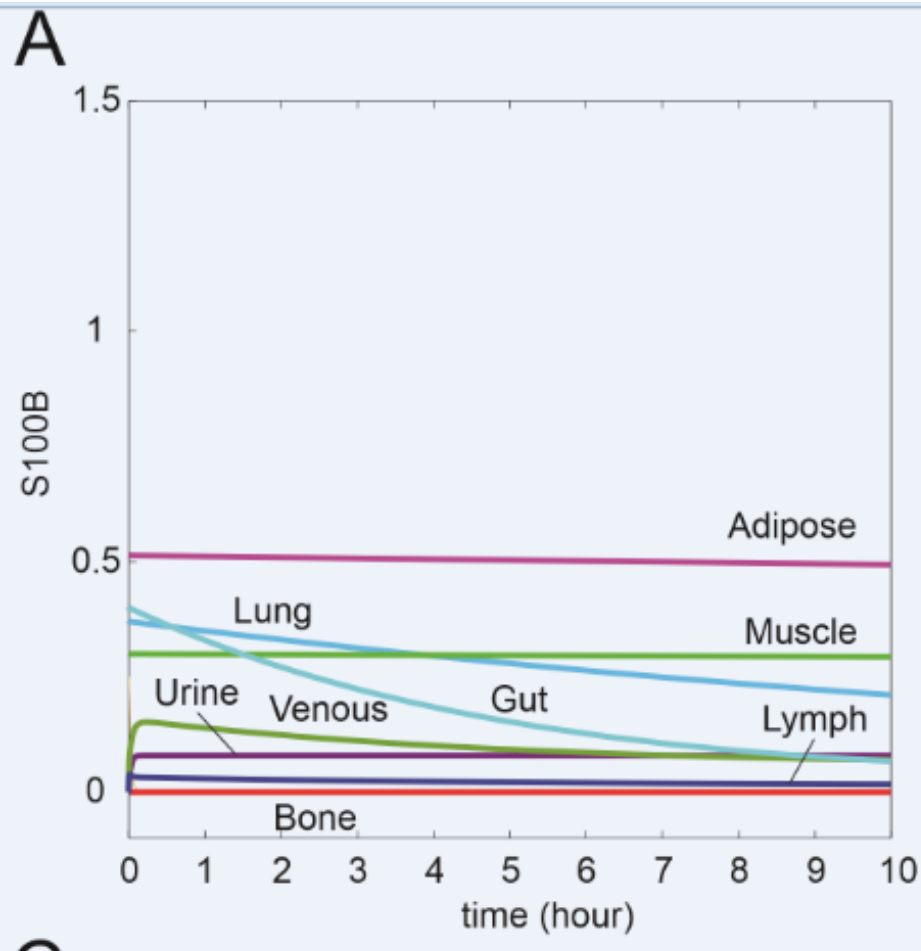

B

C
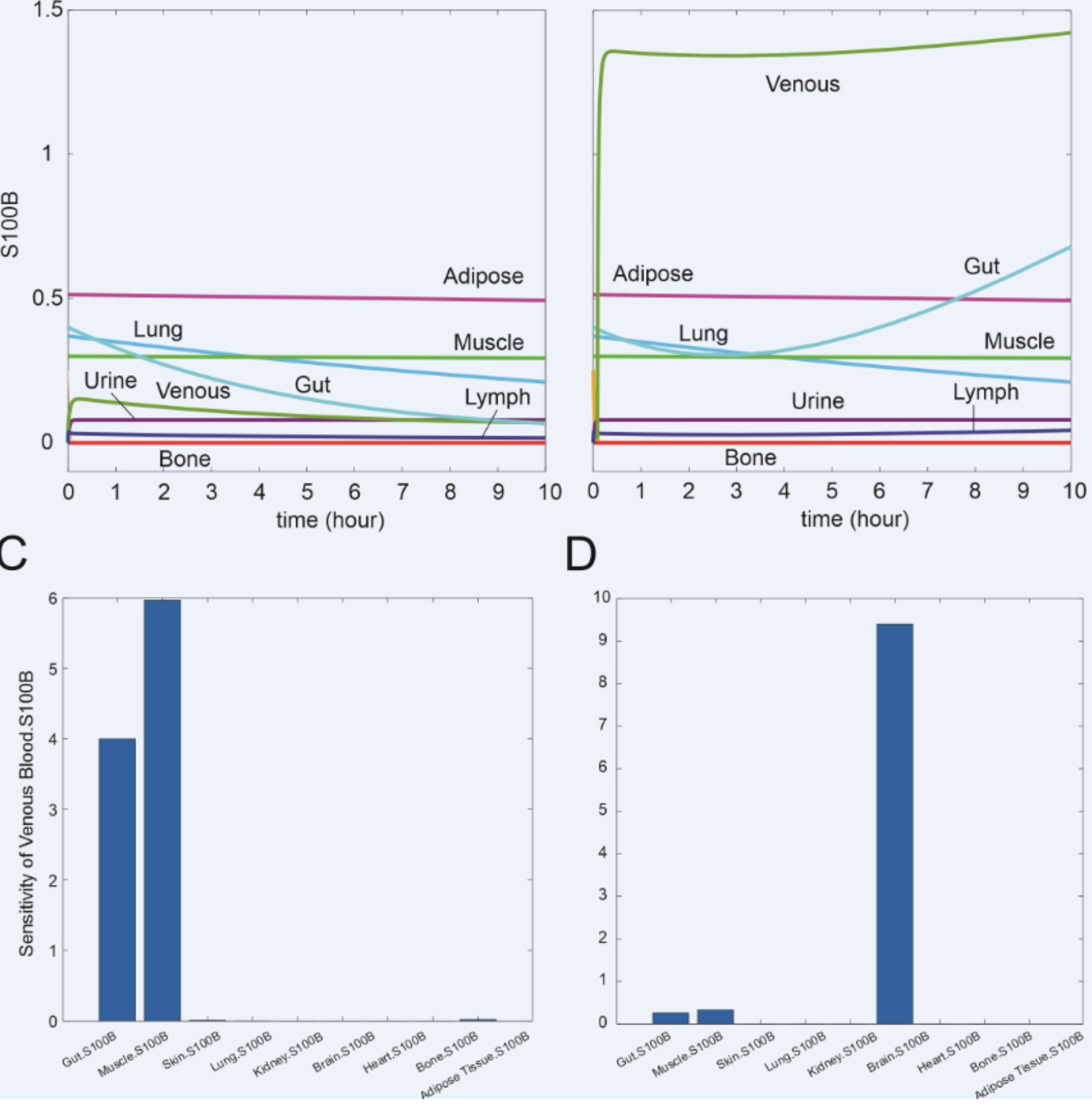

$\mathrm{D}$

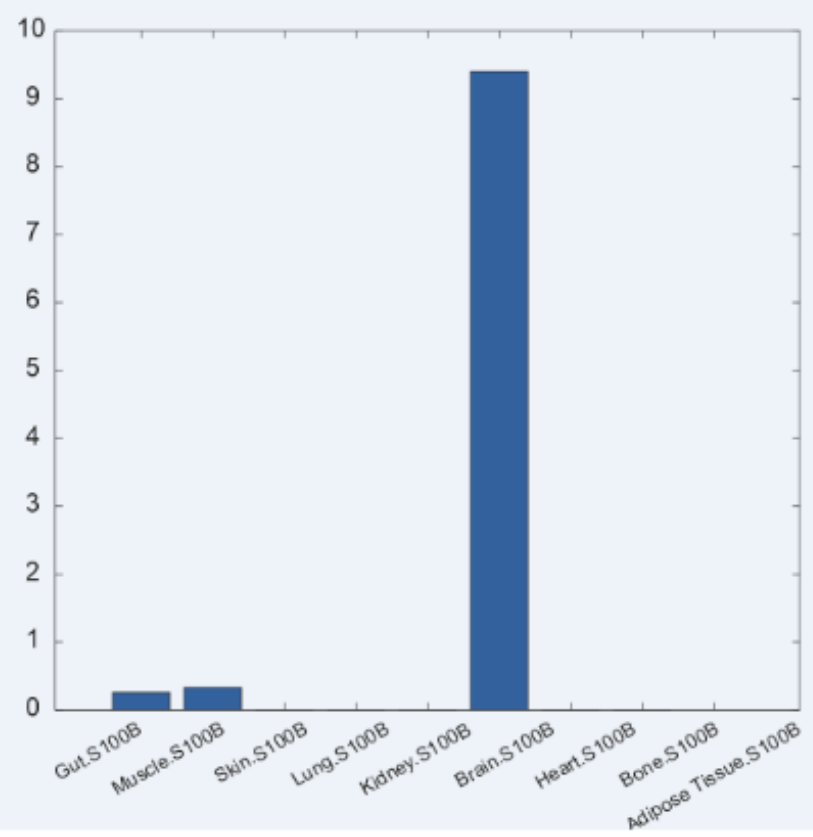

Figure 3

Results from simulations and sensitivity analysis (Model 1). The left panel shows the actual levels of S100B in organs before (A) and after BBB disruption (B) obtained by setting the BBB_Index value to 1 . Figures $C$ and $D$ show the results of sensitivity analysis queries under the same conditions. Note that before BBBD (A) venous levels at steady state never reached the $0.1 \mathrm{ng} / \mathrm{ml}$ thresholds, the upper ceiling for control values of S100B. 


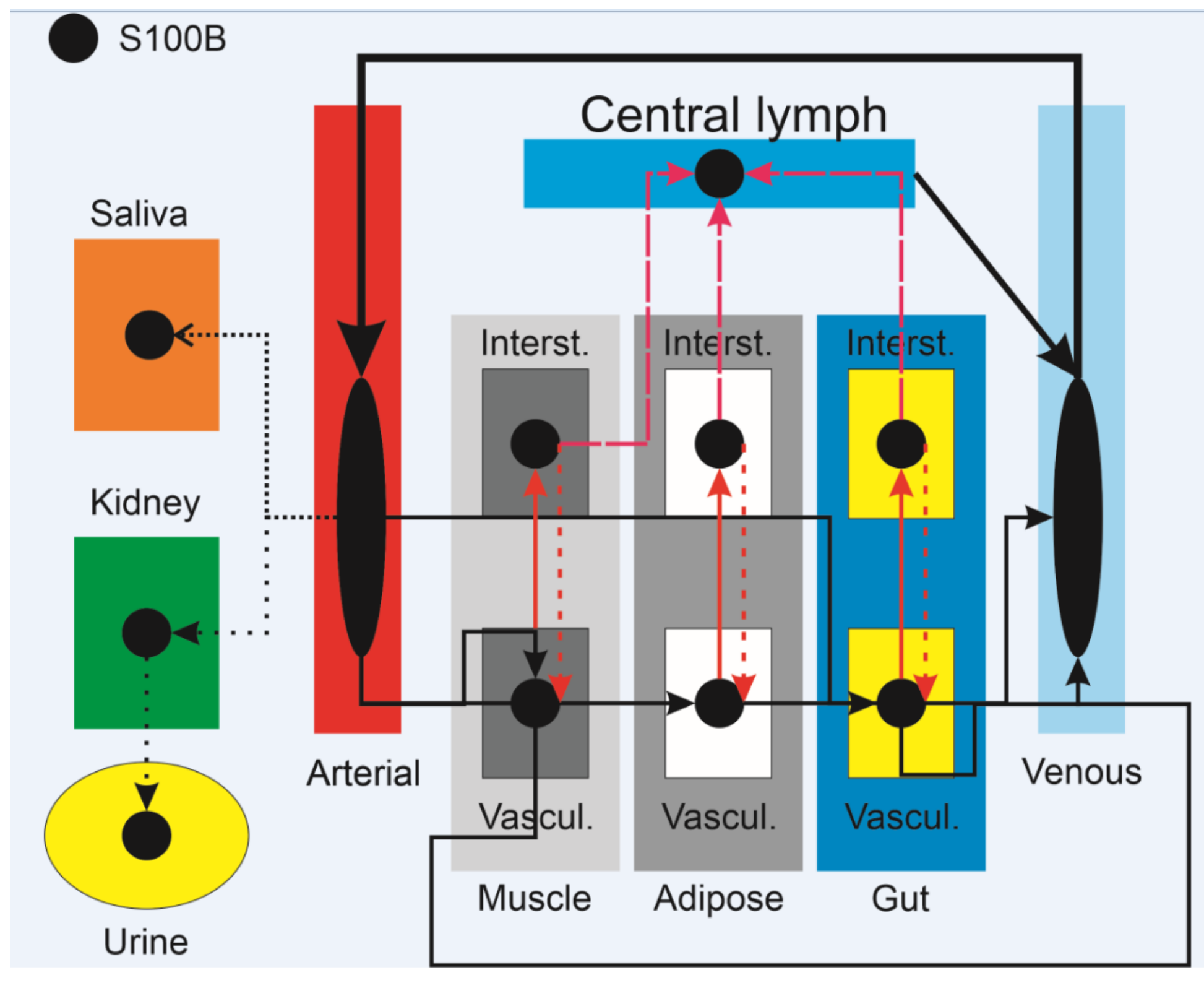

Figure 4

Schematic representation of Model 2. Note the addition of a salivary and glymphatic component. Organs are subdivided into interstitial and vascular compartments, while the brain is represented by three compartments, see Figure 6 . 
A

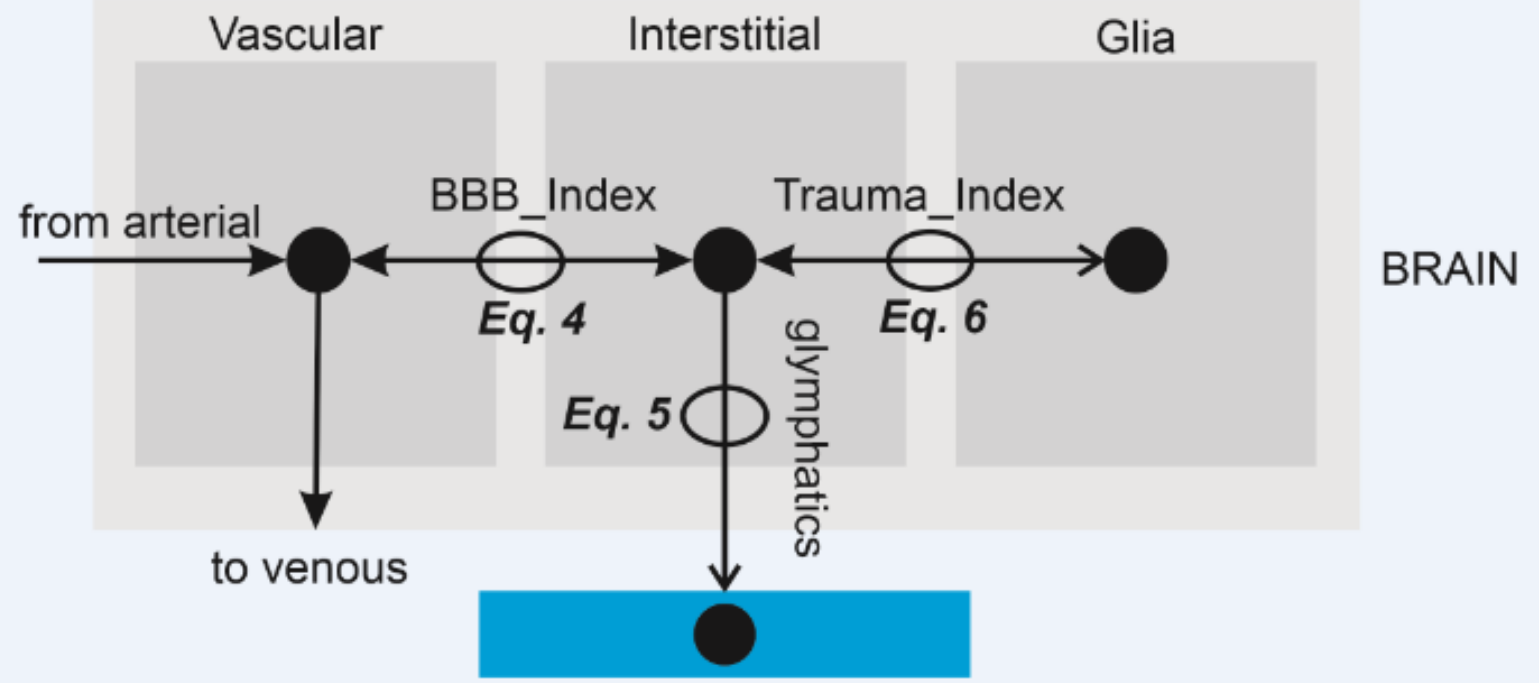

Central lymph

B

Vascular endothelial pores Small brain capillary pores Vascular endothelial pores

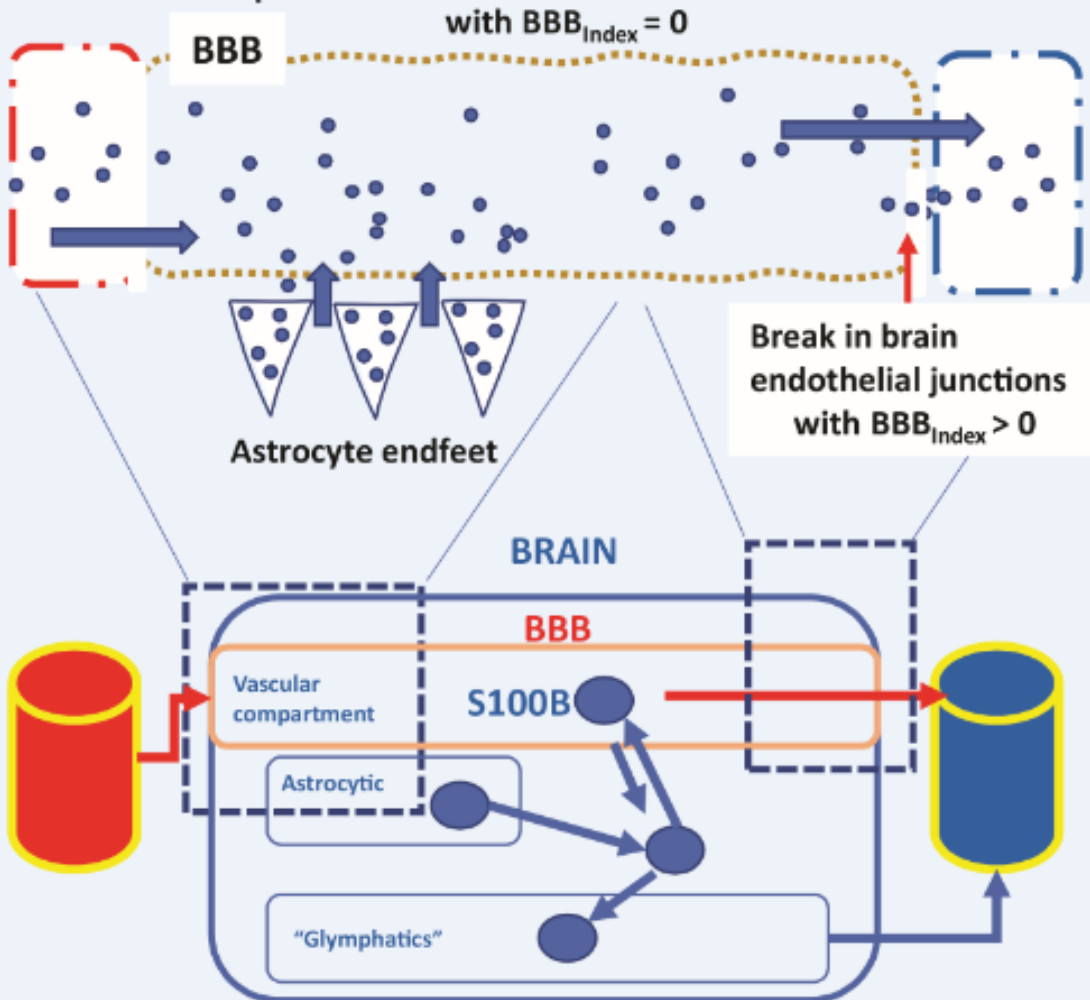

Figure 5

A) Modeling of the CNS and its communications with the periphery. B) Representation of the physiological reality to be modeled and schematics of the CNS components of the model. 


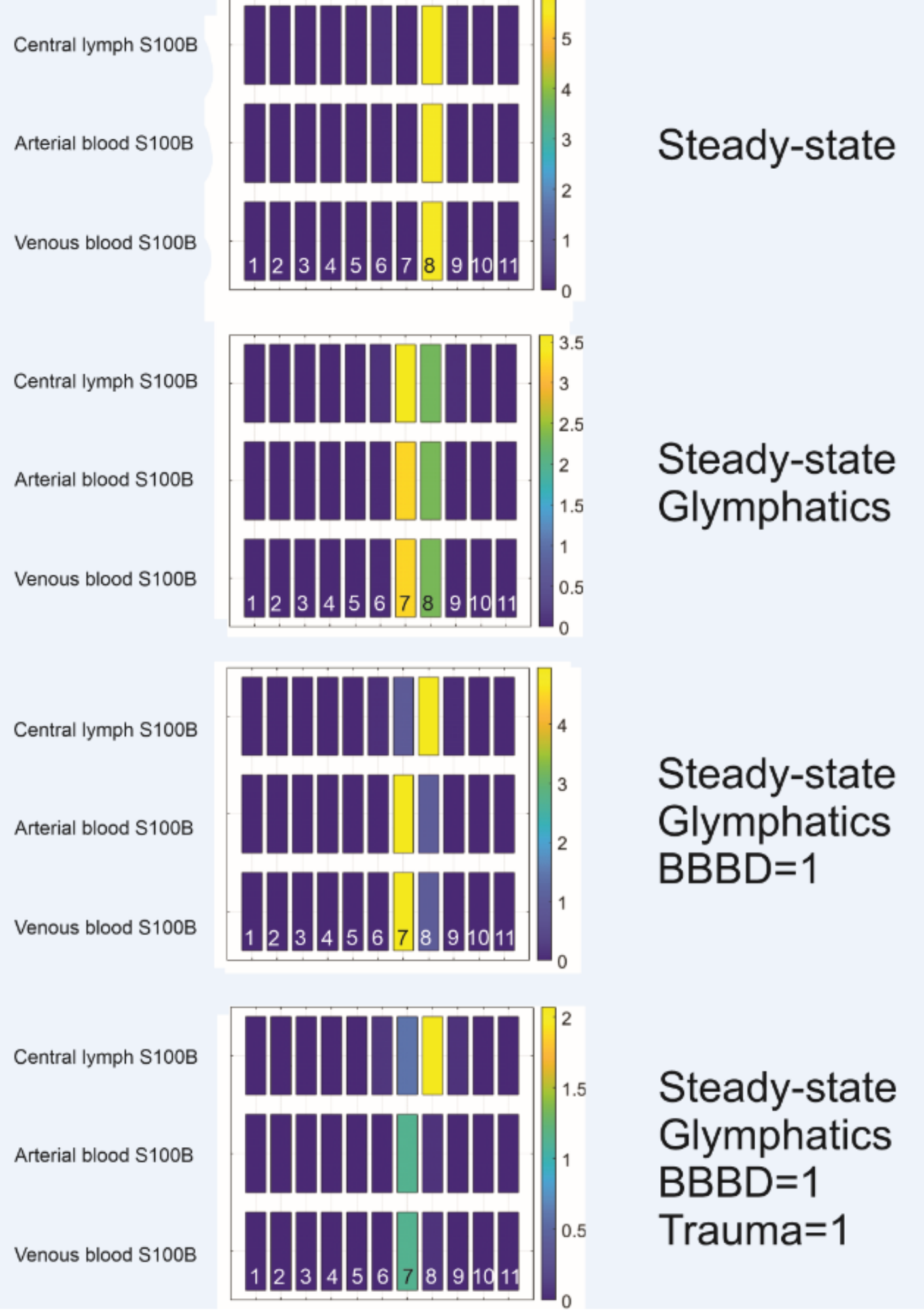

\section{Figure 6}

Sensitivity analysis for Model 2. Steady-state conditions refer to the sensitivity measured at 10 hours of simulation. The right-hand scale shows a color mapping of the results instead of numerical values. See text for details. The key for the numbers at the bottom of each panel is 1: Venous blood; 2: Vascular compartment brain; 3: Vascular compartment adipose; 4: Vascular compartment gut; 5: Vascular compartment muscle; 6: Interstitium adipose; 7: Interstitium brain; 8: Interstitium gut; 9: Interstitium 
muscle; 10: Arterial blood; 11: Central lymph. When the brain is isolated from the periphery, and the only source of S100B available is the content of peripheral organs (top panel), the gut is the chief controller of body fluids S100B. However, when a communication line between brain to the periphery is established via glymphatic drainage, the brain becomes the most influential organ for circulating S100B. This remains true after BBBD and the opening of the communication between the astrocyte content of S100B and the interstitium in the brain.
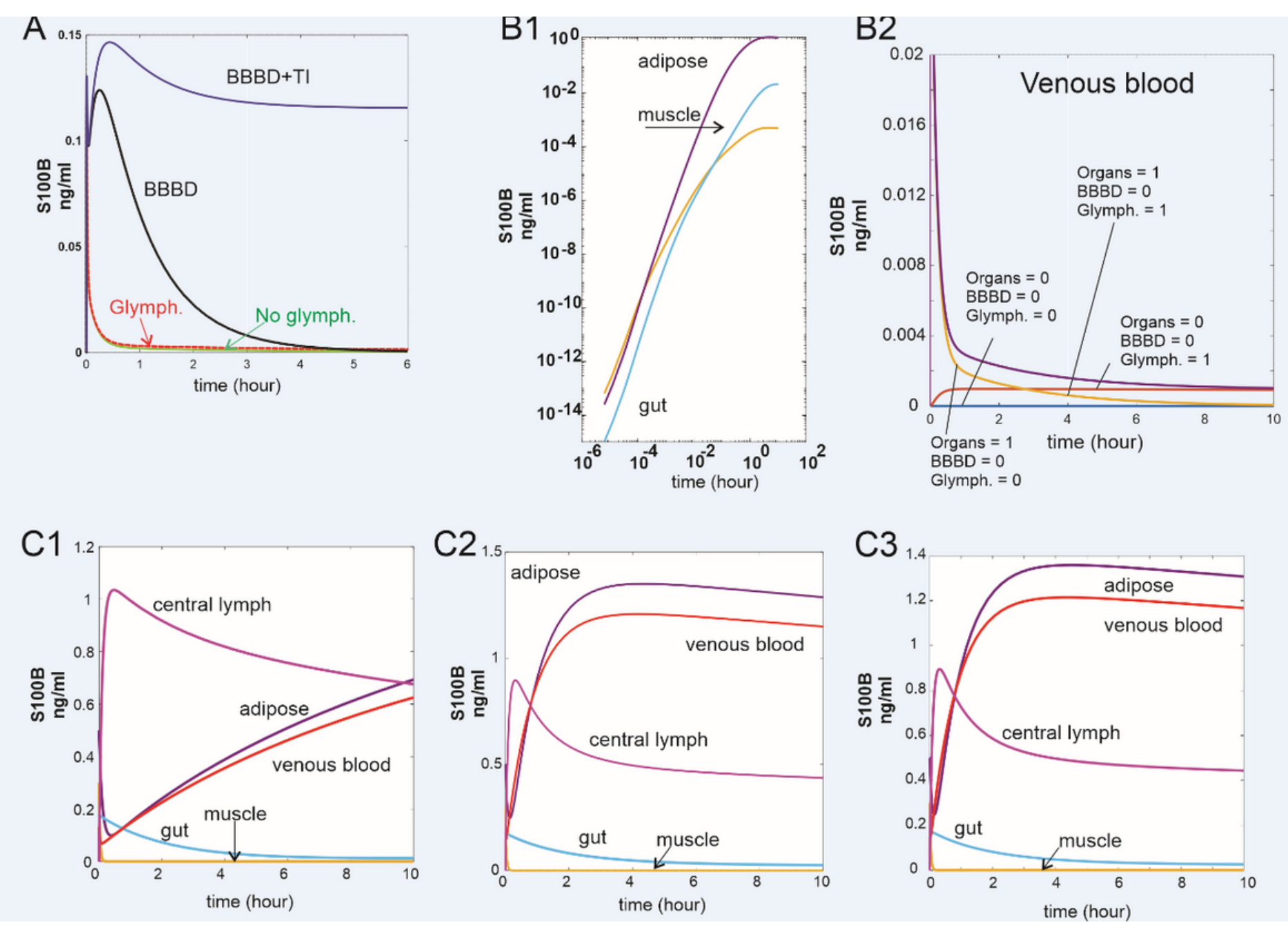

\section{Figure 7}

A) Effects of glymphatics, BBB disruption, and the trauma index on venous blood S100B. Note that activating release from astrocytes (Trauma_Index) prevents return of S100B to pre-BBBD values, suggesting that parenchymal S100B is involved in the half-life of S100B. B1 and B2). "Filling" of empty organs (S100B set at 0 in adipose, muscle, and gut tissues) after a 100-hour simulation. Changes in venous S100B in empty or full organs in the presence or absence of glymphatic contribution. The data show that peripheral levels in organs can derive from brain reservoirs. C) Time course of S100B changes in various compartments under normal (C1), BBB disruption (C2), and trauma (C3). 

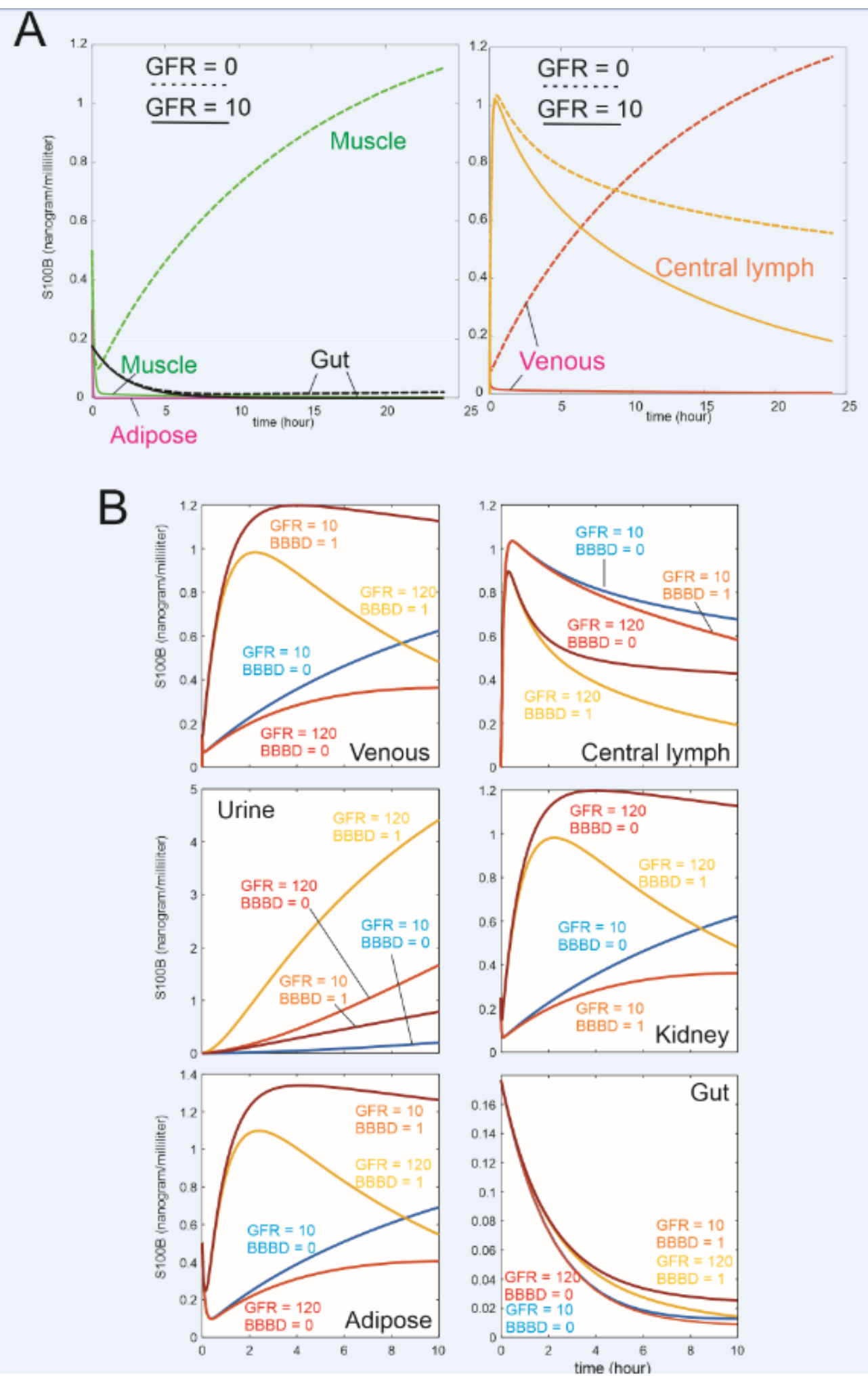

\section{Figure 8}

A) Effects of removing kidney filtration from the model. Note the increase in muscle S100B and venous levels, showing that GFR controls peripheral levels and kinetic behavior of S100B. B) Effect of varying glomerular filtration on S100B. Note the drop of venous S100B with increased glomerular filtration rate and the lack of effect of GFR on brain interstitial levels and gut S100B. Also note organ-dependent changes in S100B with low or high GFR. 

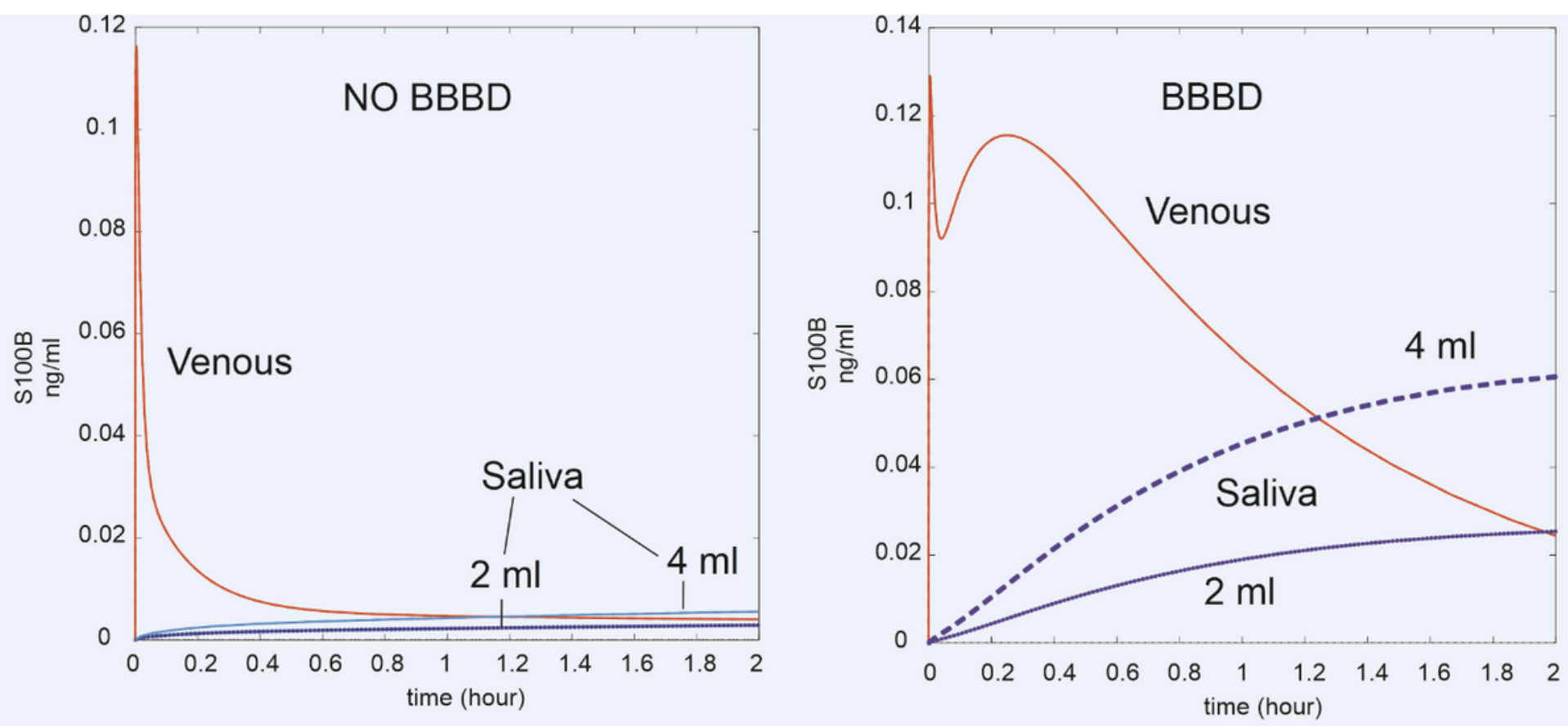

Figure 9

Salivary levels in control or after BBB disruption.

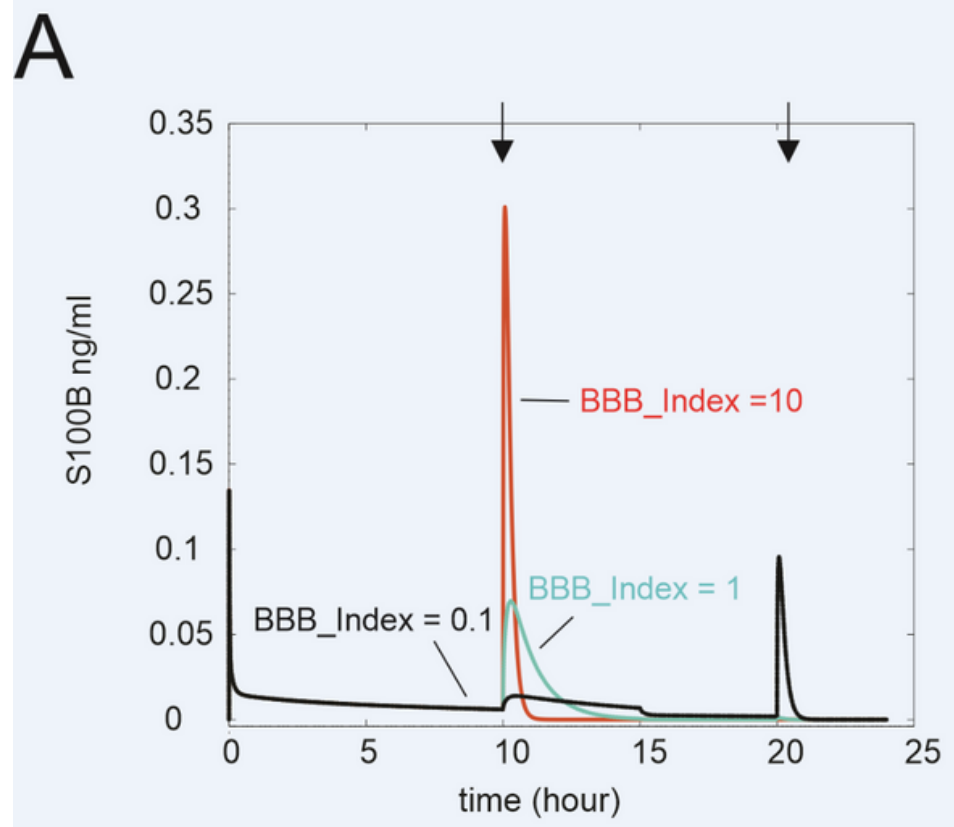

B

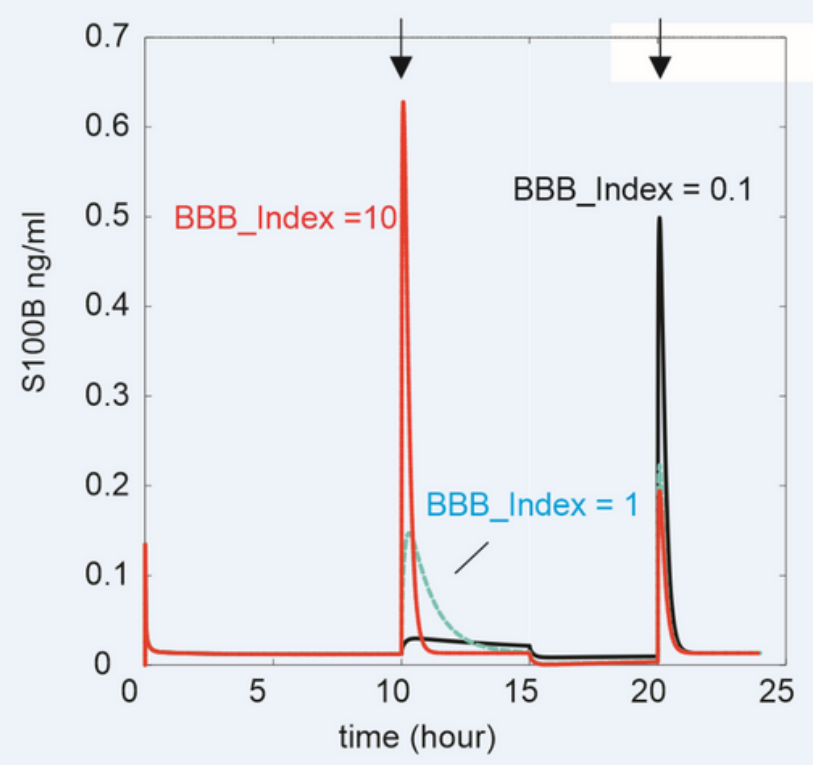

Figure 10

Effect of the previous extent of BBB disruption on secondary BBB insults. See Results and Discussion.

\section{Supplementary Files}

This is a list of supplementary files associated with this preprint. Click to download. 
- Table1.xlsx

- Table2.xlsx

- SupplementalTable1Repaired.xIsx

- SupplementalTable2Repaired.xlsx 